\title{
Integration of a Hard X-ray Microprobe with a Diffractometer for Microdiffraction
}

\author{
J. Libera ${ }^{\text {a }}$, Z. Cai ${ }^{*}$, B. Lai and S. Xu \\ Advanced Photon Source, Argonne National Laboratory, Argonne, IL 60439 \\ ${ }^{a}$ Advanced Photon Source, Argonne National Laboratory and Northwestern University, \\ Materials Science and Engineering, Evanston IL, 60608
}

\begin{abstract}
A hard x-ray Fresnel zone-plate-based microprobe has been designed and integrated into a Newport kappa diffractometer for microdiffraction studies at the 2ID-D beamline at the Advance Photon Source. The microprobe employs $10 \mathrm{~cm}$ and $40 \mathrm{~cm}$ (focal length at $8 \mathrm{keV}$ ) zone plates to provide high and moderate focusing power, respectively. Each zone-plate assembly has two identical zone plates stacked together to provide higher focusing efficiency for higher energy (30 keV) applications. The mounting base of the microprobe is supported by the side of the base of the diffractometer at one end and the central table of the diffractometer at the other end, thus minimizing the instability of relative positions between the focal spot and the specimen. A x-ray focal spot size smaller than $360 \mathrm{~nm}$ and angular repeatability of the sample circles smaller than $0.001 \mathrm{deg}$. have been demonstrated with this setup.
\end{abstract}

\footnotetext{
* To whom correspondence should be addressed. E-mail: cai@aps.anl.gov
} 
A zone-plate-based hard x-ray microprobe (HXRM) has been implemented for a wide range of $\mathrm{x}$-ray experimental techniques ${ }^{1}$ at station 2ID-D of the Advanced Photon Source (APS) at Argonne National Laboratory. We report here the integration of a HXRM with a six-circle diffractometer for microdiffraction experiments. The kappa geometry of the diffractometer was selected to accommodate the needs of the HXRM for the space near the specimen and, thus, to provide a wider access to reciprocal space. The HXRM uses the radiation generated by the APS undulator A. ${ }^{2}$ Windowless operation (except the beam exit windows) and the first optical component of a water-cooled, grazing-incidence, horizontal-deflection mirror were designed to preserve source brilliance and beam coherence and to suppress high-order harmonics of the undulator radiation. The energy of the x-rays is selected with a double-crystal $\operatorname{Si}(111)$ monochromator. The selectivity of the horizontal coherence length of the beam is achieved by a white-beam slit located 43 meters upstream of the HXRM.

The diffractometer is an N6050-K1 goniometer made by Newport Micro-Controle with four sample circles and two detector circles. The four sample circles are in the kappa geometry with a $50^{\circ}$ angle between the kappa and omega axes. The on-axis encoders (Heidenhain's RON 287 and ERA 180) of the closed-loop servodriver systems provide angular resolution of $0.0001^{\circ}$ for sample and detector circles and $0.00025^{\circ}$ for circles that carry the sample-circle assembly and detector-arm assembly. In order to characterize the repeatability of the angular motion, the (400) reflection from a silicon wafer was measured with a $10.2 \mathrm{keV}$ x-ray beam of size $1 \mathrm{~mm}^{2}$. The results obtained by rocking the phi axis with the detector slits wide opened are shown in Fig. 1. Although the peak was broadened by the energy spreading of the x-rays $\left(\delta \mathrm{E} / \mathrm{E} \sim 10^{-4}\right)$ and the mosaicity of the silicon wafer (typically $.003^{\circ}-.005^{\circ}$ ), angular profiles in $0.0002^{\circ}$ steps from 
consecutive scans are overlapped within an angular range less than $0.001^{\circ}$. Similar results were obtained from other circles.

The HXRM employs gold phase zone plates with focal lengths of $10 \mathrm{~cm}$ and $40 \mathrm{~cm}$ (at 8 $\mathrm{keV})$ to achieve high and moderate focusing power, respectively. An order-sorting aperture (OSA) of $20 \mu \mathrm{m}$ is used to select the first-order focusing beam, and a gold dot $30 \mu \mathrm{m}$ in diameter and $40 \mu \mathrm{m}$ thick is used to reduce the zero-order beam through the $20 \mu \mathrm{m}$ OSA. The $10 \mathrm{~cm}$ zone plate has a diameter of $150 \mu \mathrm{m}$, an outmost zone width of $0.1 \mu \mathrm{m}$, and a focal efficiency of $10 \%$ $(10 \mathrm{keV}),{ }^{3}$ the $40 \mathrm{~cm}$ zone plate has a diameter of $250 \mu \mathrm{m}$, an outmost zone width of $0.25 \mu \mathrm{m}$, and a focal efficiency of $25 \%(10 \mathrm{keV})$. Photon fluxes of $1 \times 10^{10}$ photons $/ \mathrm{sec} / 0.01 \% \mathrm{BW}$ and $1.1 \times 10^{11}$ photons $/ \mathrm{sec} / 0.01 \% \mathrm{BW}$ have been measured at the focal spots generated by the $10 \mathrm{~cm}$ and $40 \mathrm{~cm}$ zone plates, respectively, corresponding to photon density gains of 15,000 and 8,000 . Circular focal spots can be obtained using the white-beam slits (horizontal) with photon fluxes one order of magnitude less. The size and the focal length of the zone plate determine the angular divergence (divergence=zone plate size/focal length).

In order to further improve focusing efficiency and extend the zone-plate microfocusing capability into higher energy, a two-zone-plate stacking technique can be implemented in the HXRM. Two identical zone plates are positioned within the focal depth $(100 \mu \mathrm{m}$ for $10 \mathrm{~cm}$ zone plate) longitudinally and within the outmost zone width $(0.1 \mu \mathrm{m}$ for $10 \mathrm{~cm}$ zone plate) transversely, equivalently doubling the thickness of a single zone plate. Efficiency gains as high as 3.5 times have been measured with $10 \mathrm{~cm}$ zone plates at $10 \mathrm{keV}$, and a focusing efficiency better than $12 \%$ is expected at $30 \mathrm{keV} .{ }^{4}$ Two zone plates are carried by the $\mathrm{XYZ}$ motorized stages for focal length adjustment and positioning in the transverse plane, and one of the two zone plates can be driven by a XY piezostage for alignment against the other. The criteria for 
two zone plates to be aligned have been established by looking at the interference patterns of xrays focused by individual zone plates. In Fig. 2 we display the patterns obtained with different extents of the displacement between the two zone plates recorded at a $\mathrm{CdWO}_{4}$ scintillation screen placed downstream of the focal spot. Decreasing the spatial frequency of the interference feature in the pattern indicates a good trend in the alignment, and the spatial frequency becomes zero as the two zone plates are perfectly aligned. However, one has to avoid the other extreme where the two zone plates are completely off aligned and no interference would take place.

The stability of the relative positions between the focal spot, which is defined by the zone-plate optical axis, and the sample, which is held by the four-circle assembly, characterizes the performance of the instrument. The stability of the supporting structure for the HXRM assembly is critical for achieving high performance. As shown in Fig. 3, the table top for the HXRM is supported at one end directly by the central table of the diffractometer (labeled A in the figure), and the other end is supported by a L-shape weldment of structural steel (20 cm x 20 $\mathrm{cm}, 1 \mathrm{~cm}$ thick, and $15 \mathrm{~cm} \times 25 \mathrm{~cm}, 1 \mathrm{~cm}$ thick) reinforced with welded steel webs of $12 \mathrm{~mm}$ thick along both the vertical and horizontal directions (labeled B in the figure). Both the central table and the weldment are mounted on the diffractometer's stone base. Vibration measurement was performed using a pair of seismic accelerometers within a frequency range from $0.1-100$ Hz. Coupled to a gain-100 preamplifier, the accelerometer system has a sensitivity of $0.7 \mathrm{~V} / \mathrm{mg}$. A Hewlett Packard 35665A dynamic signal analyzer was applied to carry out real-time FFT analyses. In Fig.4 we display the vertical and horizontal vibration spectra from 0 to $100 \mathrm{~Hz}$ measured at the zone-plate assembly of the HXRM. Two major peaks appear in the vertical spectrum at 8 and $18 \mathrm{~Hz}$ with amplitudes of 33 and $52 \mathrm{~nm} / \mathrm{Hz}^{1 / 2}$, and one major peak appears in 
the horizontal spectrum at $8 \mathrm{~Hz}$ with amplitude of $150 \mathrm{~nm} / \mathrm{Hz}^{1 / 2}$. These results are similar to those measured at the stone base of the diffractometer, indicating no amplification of the vibration is generated because of the supporting structure.

The size of the focal spot was measured with a $10 \mathrm{~cm}$ zone plate and $10 \mathrm{keV}$ x-rays using a 20-nm-thick Cr knife-edge. The sample stage assembly that carries the $\mathrm{Cr}$ knife-edge is mounted on sample circles of the diffractometer. The intensity profile of the $\mathrm{Cr} \mathrm{K}$ line fluorescence obtained by scanning the knife-edge across the focal spot was fitted to a sum of an error function and a linear background. As shown in Fig. 5, a FWHM size of $359 \mathrm{~nm}$ was obtained along the vertical direction. The measured spot size results from a convolution of the beam size, vibration amplitude, and the quality of the $\mathrm{Cr}$ edge. The knife-edge was obtained by breaking a Cr-coated silicon wafer at liquid nitrogen temperature without further examination. The quality of the knife-edge probably limited the measured spot size. Efforts are currently underway to achieve a smaller spot size by replacing the knife-edge with a nanowire and attaining better isolation from the floor vibrations. A similar effort was successfully made for another microdiffraction setup at the 2ID-D station. ${ }^{5}$

This work was supported by the U.S. Department of Energy, Office of Science, under Contract No. W-31-109-Eng-38.

\section{$\underline{\text { References: }}$}

1. Z. Cai, B. Lai, W. Yun, P. Ilinski, D. Legnini, J. Maser, W. Rodrigues, X-ray

Microscopy, Proceedings of the Sixth International Conference, Vol. 507, W. Meyer-Ilse,

T. Warwick, and D. Attwood, eds., (AIP, 2000) pp. 472-477. 
2. R. J. Dejus, B. Lai, E. R. Moog, E. Gluskin, Argonne National Laboratory Report No. ANL/APS/TB-17, May 1994.

3. W. Yun, B. Lai, Z. Cai, J. Maser, D. Legnini, E. Gluskin, Z. Chen, A. A. Krasnoperova, Y. Vladimirsky, F. Cerrina, E. Di Fabrizio, and M. Gentili, Rev. Sci. Instrum., 70(5), 2238 (1999).

4. Shastri, S. D., Maser, J. M., Lai, B., Tys, J., Optics Communications, 197(1), 9 (2001).

5. Xu, S. L., Lai, B., Cai, Z., Shu, D., "Vibration-Damping Structure Design for a X-ray Microprobe Supporting System", this proceeding.

\section{Figure Captions}

1. Intensity profiles of four consecutive scans of the $\mathrm{Si}(400)$ reflection measured at $10.2 \mathrm{keV}$ from a silicon wafer using the phi circle.

2. Images of interference patterns formed from x-rays focused with two zone plates, recorded downstream from the focal plane. Image sequence shows the alignment procedure of two stacked zone plates. The dark spot in the center is due to the beam stop.

3. Diffractometer and the HXRM in the 2ID-D station of the APS. X-ray beam enters from the left through an evacuated beam pipe.

4. Horizontal (bold line) and vertical (thin line) vibration spectra measured on the zone-plate assembly of the HXRM.

5. Cr fluorescence intensity profile (dots) measured while scanning a $\mathrm{Cr}$ knife-edge across the focal spot, fit to the measured curve (solid line), and the derivative of the fitted curve (dashed line). 
Figure 1

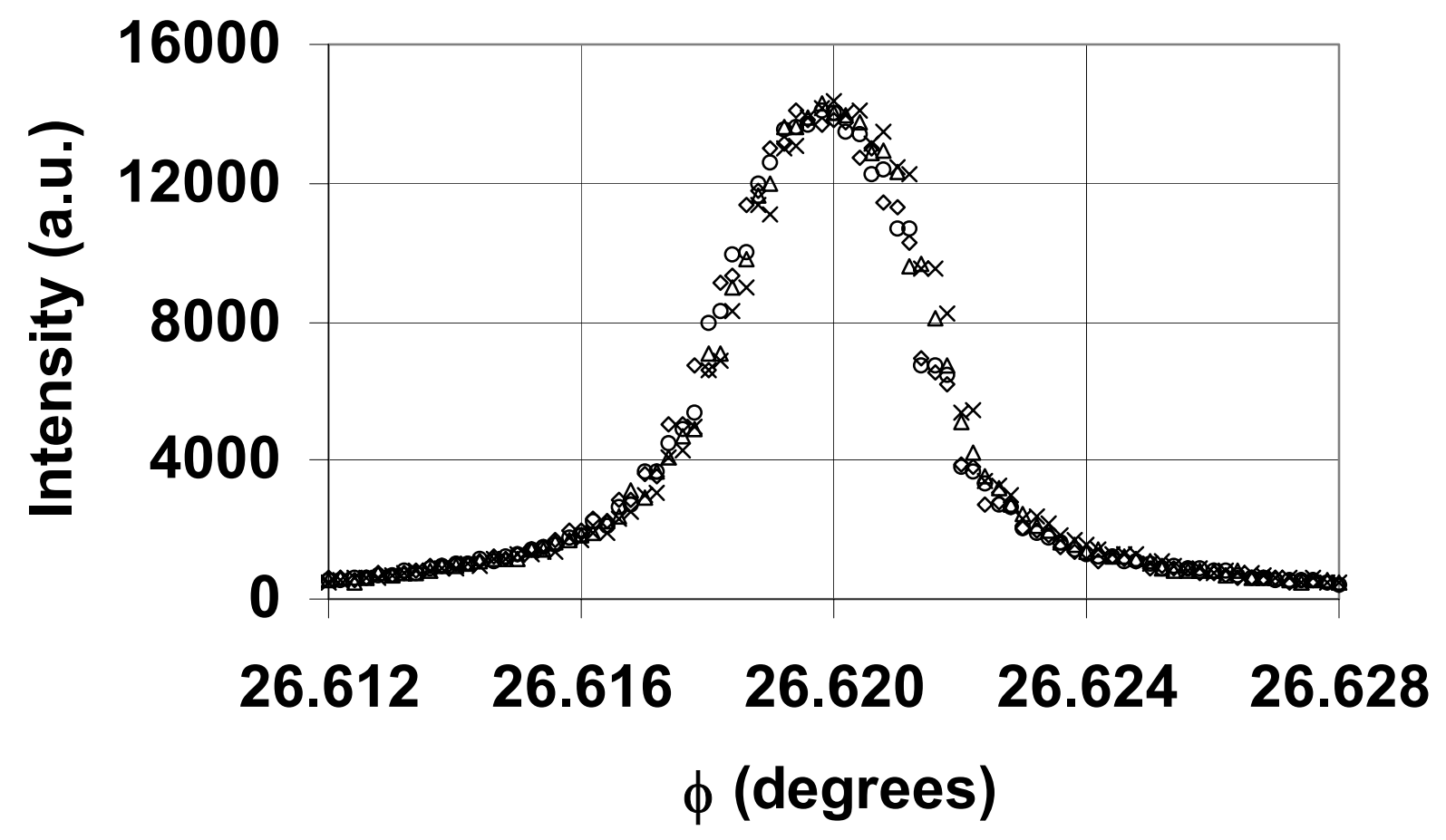


Figure 2

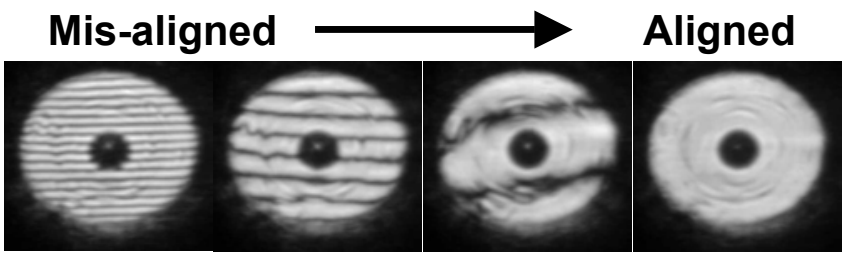


Figure 3

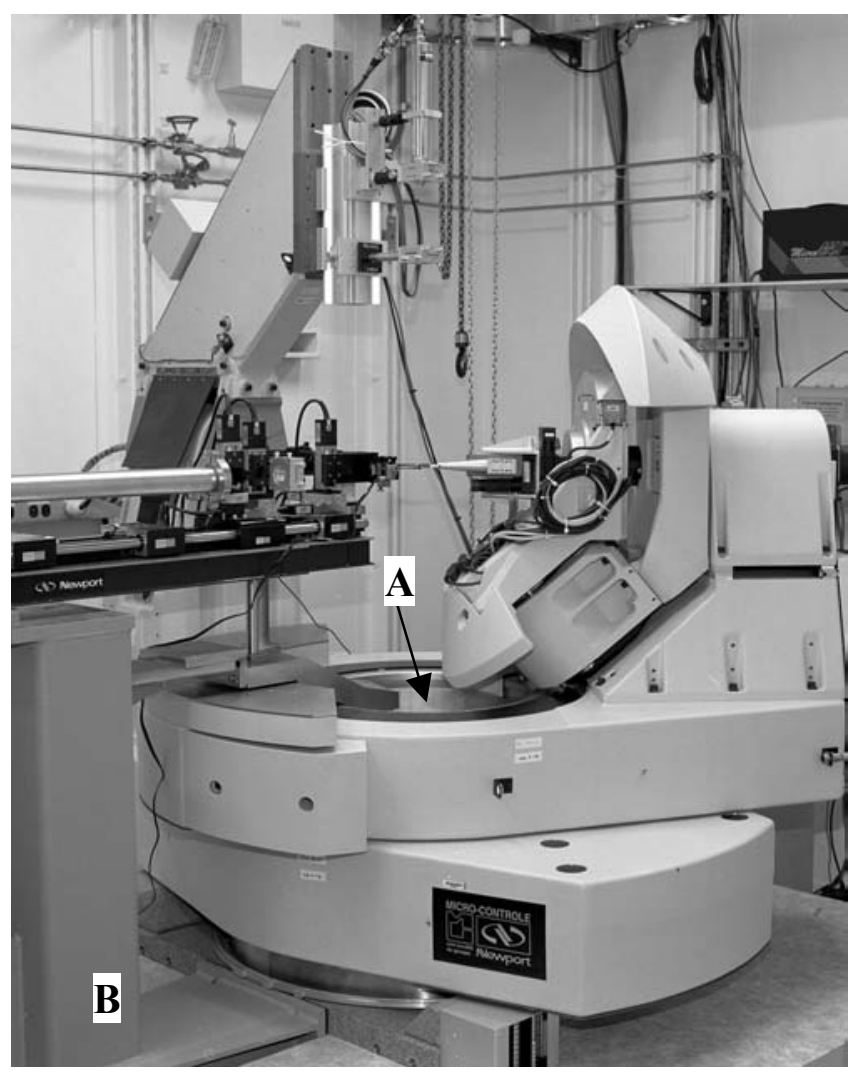


Figure 4

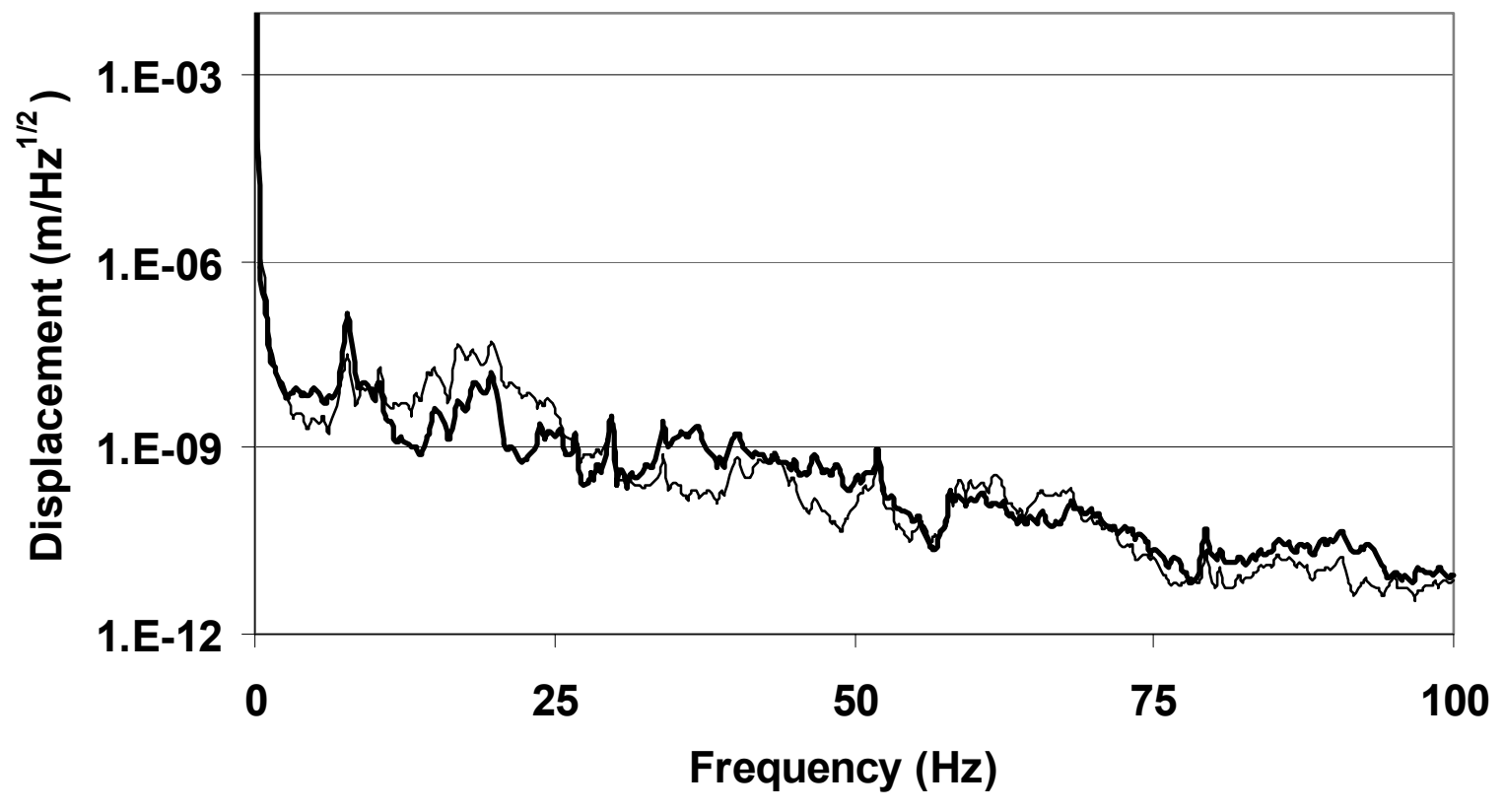


Figure 5

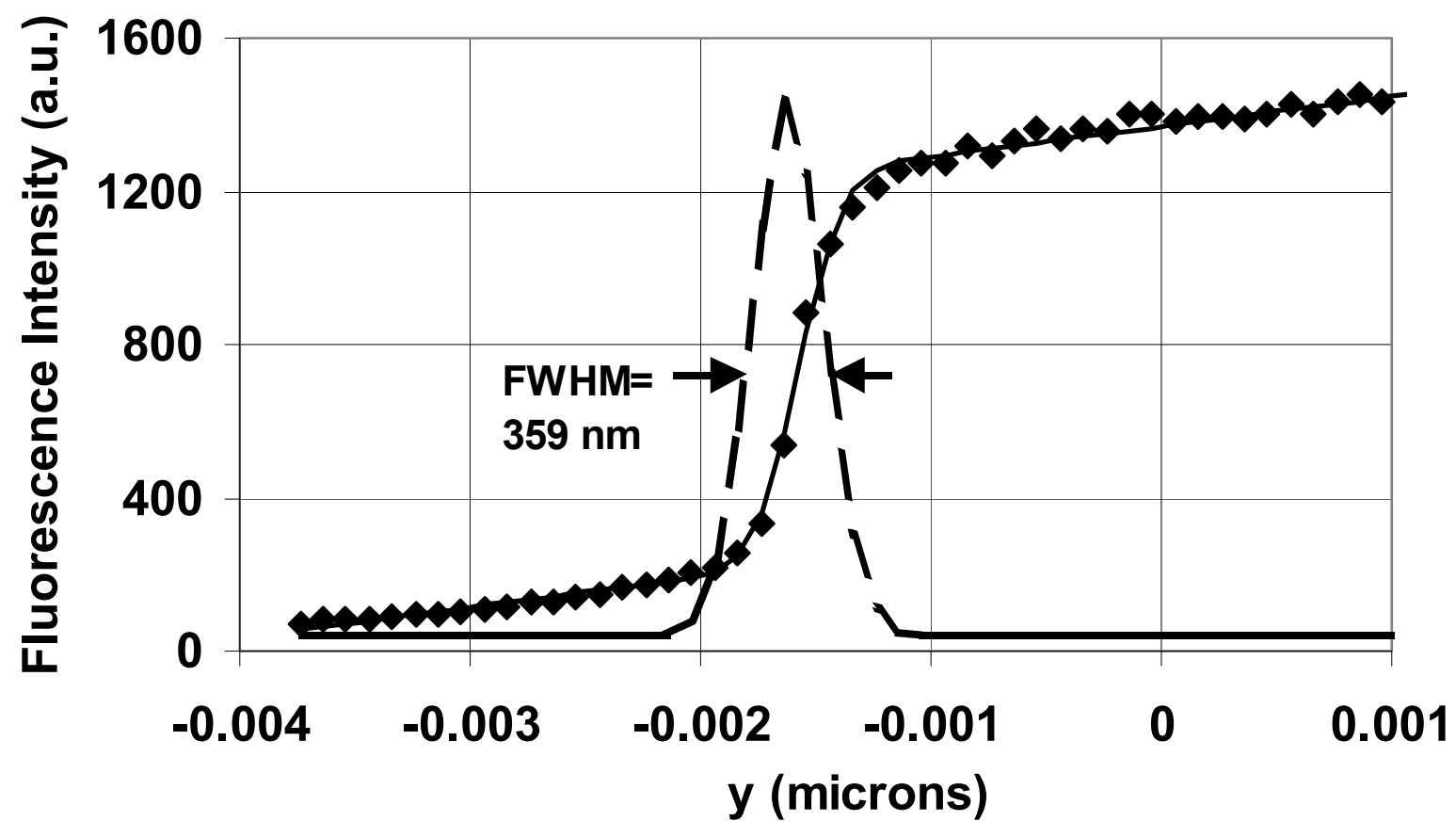




\section{Figure Captions}

1. Microdiffraction experiment at beamline 2ID-D of the APS, x-ray beam enters from the left through an evacuated beam pipe.

2. Schematic representation of diffractometer and laboratory reference frame.

3. Image sequence showing the alignment procedure of two stacked zone plates.

4. Four consecutive scans of the as prepared sample using $\phi$ axis

5. Focal spot measurement using Cr knife-edge, Cr fluorescence intensity (dots), error function curve fit (solid line), derivative of the error function (dashed line)

6. Horizontal (thin line) and vertical (bold line) vibration amplitude measurement on the microbeam optics table. 